PROCEEDINGS OF THE

AMERICAN MATHEMATICAL SOCIETY

Volume 136, Number 12, December 2008, Pages 4467-4473

S 0002-9939(08)09425-2

Article electronically published on June 17, 2008

\title{
REFLEXIVITY AND SETS OF FRÉCHET SUBDIFFERENTIABILITY
}

\author{
ONDŘEJ KURKA
}

(Communicated by N. Tomczak-Jaegermann)

\begin{abstract}
We show that the sets of Fréchet subdifferentiability of Lipschitz functions on a Banach space $X$ are Borel if and only if $X$ is reflexive. This answers a question of L. Zajíček.
\end{abstract}

\section{InTRODUCTION AND MAIN RESULT}

Let $X$ be a real normed linear space and $f$ be a real function on $X$. Let $x \in X$. We say that $u \in X^{*}$ is a Fréchet subgradient of $f$ at $x$ if

$$
\liminf _{y \rightarrow x} \frac{f(y)-f(x)-u(y-x)}{\|y-x\|} \geq 0 .
$$

The set of all Fréchet subgradients of $f$ at $x$ is called the Fréchet subdifferential of $f$ at $x$ and denoted by $\partial f(x)$. The set of all points $x \in X$ at which $\partial f(x) \neq \emptyset$ is called the set of Fréchet subdifferentiability and denoted by $S(f)$.

Further on, we omit "Fréchet" in the above notions and we suppose that all normed linear spaces are real.

At first, we recall some known results about the sets of subdifferentiability.

Theorem 1.1 ([, Section 4]). Let $f$ be a lower semicontinuous function on a normed linear space $X$. Then $S(f)$ is a Suslin set.

We recall the definition of a Suslin set in Section 2.

L. Zajíček posed in [6, Section 4] the question of whether $S(f)$ must be Borel for every lower semicontinuous function. We show in Theorem 1.3 below that the answer to Zajíček's question is negative in non-reflexive spaces. The situation in the reflexive case was clarified by an unpublished remark of P. Holický and M. Laczkovich. A proof of their result will be given at the end of this section.

Theorem 1.2 (Holický, Laczkovich). Let $f$ be a lower semicontinuous function on a normed linear space $X$ with a reflexive completion. Then $S(f)$ is an $F_{\sigma \delta \sigma}$ set.

We note that there is a continuous function $f$ on $\mathbb{R}^{3}$ such that $S(f)$ is not $G_{\delta \sigma \delta}$ (see [3]). We now formulate the main result. Its proof will be given in Section 2.

Theorem 1.3. Let $X$ be a normed linear space with a non-reflexive completion. Then there is a Lipschitz function $f$ on $X$ such that $S(f)$ is not Borel.

Received by the editors April 5, 2007, and, in revised form, November 1, 2007.

2000 Mathematics Subject Classification. Primary 54H05, 46B10, 46 G05.

Key words and phrases. Reflexivity, set of Fréchet subdifferentiability, Borel set, Suslin set.

(C)2008 American Mathematical Society 
Remark 1.4. Theorem 1.1 can be generalized. M. Šmídek has proved that Theorem 1.1 holds for Borel functions (see [5]). It follows from his method and Theorem 1.2 that $S(f)$ is Borel if $f$ is a Borel function on a space with a reflexive completion.

Proof of Theorem [1.2. By [6, Lemma 4], the set

$A_{n_{1}, \ldots, n_{k}}^{K}=\bigcup_{\|u\| \leq K} \bigcap_{i=1}^{k}\left\{x \in X:\|y-x\|<\frac{1}{n_{i}} \Rightarrow f(y)-f(x) \geq u(y-x)-\frac{1}{i}\|y-x\|\right\}$

is closed for $K, k, n_{1}, \ldots, n_{k} \in \mathbb{N}$. It is enough to verify that

$$
S(f)=\bigcup_{K=1}^{\infty} \bigcap_{k=1}^{\infty} \bigcup_{\left(n_{1}, \ldots, n_{k}\right) \in \mathbb{N}^{k}} A_{n_{1}, \ldots, n_{k}}^{K} .
$$

Let $x \in S(f)$. There exists $u \in \partial f(x)$. For some $K \in \mathbb{N}, K \geq\|u\|$. By the definition of the subgradient, for every $i \in \mathbb{N}$, there exists $n_{i} \in \mathbb{N}$ such that $\|y-x\|<$ $\frac{1}{n_{i}} \Rightarrow f(y)-f(x) \geq u(y-x)-\frac{1}{i}\|y-x\|$. Now, $x \in A_{n_{1}, \ldots, n_{k}}^{K}$ for every $k \in \mathbb{N}$, which gives the inclusion " $\subset$ ". To prove the other inclusion, suppose that $K \in \mathbb{N}$ and $x \in \bigcap_{k=1}^{\infty} \bigcup_{\left(n_{1}, \ldots, n_{k}\right) \in \mathbb{N}^{k}} A_{n_{1}, \ldots, n_{k}}^{K}$. For every $k \in \mathbb{N}$, there exist $n_{k} \in \mathbb{N}$ and $u \in X^{*},\|u\| \leq K$, such that $\|y-x\|<\frac{1}{n_{k}} \Rightarrow f(y)-f(x) \geq u(y-x)-\frac{1}{k}\|y-x\|$. Consequently, for every $k \in \mathbb{N}$, the set

$$
C_{k}=\left\{u \in X^{*}:\|u\| \leq K, \liminf _{y \rightarrow x} \frac{f(y)-f(x)-u(y-x)}{\|y-x\|} \geq-\frac{1}{k}\right\}
$$

is non-empty. One can easily check that these sets are closed and convex, so they are $w$-closed, too. They are bounded at the same time. Since $X^{*}$ is reflexive, $\left\{C_{k}\right\}_{k \in \mathbb{N}}$ is a decreasing system of non-empty $w^{*}$-compact sets. Therefore, its intersection is non-empty. The easy observation that $\bigcap_{k=1}^{\infty} C_{k} \subset \partial f(x)$ completes the proof.

\section{FunCtions With NON-Borel SEts of SUbDifferentiability}

Let us recall some definitions and notation. By $\mathbb{N}<\omega$ we will denote the set of all finite sequences of natural numbers, i.e., $\mathbb{N}^{<\omega}=\{\emptyset\} \cup \bigcup_{l=1}^{\infty} \mathbb{N}^{l}$. The closed unit ball of a Banach space $X$ will be denoted by $B_{X}$. We use "co" for the convex hull, "c" for its closure and " $\overline{\mathrm{sp}}$ " for the closed linear span. Given normed linear spaces $X, Y$, we define $X \oplus_{\infty} Y$ as the sum of $X$ and $Y$ with the norm $\|(x, y)\|=$ $\max \{\|x\|,\|y\|\}, x \in X, y \in Y$. By $c$-Lipschitz we mean Lipschitz with a constant $c$.

Let $X$ be a metric space. We say that $M \subset X$ is Suslin if

$$
M=\bigcup_{\left(n_{1}, n_{2}, \ldots\right) \in \mathbb{N}^{\mathbb{N}}} \bigcap_{k=1}^{\infty} A_{n_{1}, \ldots, n_{k}},
$$

where $A_{n_{1}, \ldots, n_{k}},\left(n_{1}, \ldots, n_{k}\right) \in \mathbb{N}<\omega$, are closed in $X$. Equivalently, we may consider $A_{n_{1}, \ldots, n_{k}},\left(n_{1}, \ldots, n_{k}\right) \in \mathbb{N}<\omega$, to be open (we have $\bigcap_{k=1}^{\infty} A_{n_{1}, \ldots, n_{k}}=\bigcap_{k=1}^{\infty}\{x \in X$ : $\left.\left.\operatorname{dist}\left(x, \bigcap_{i=1}^{k} A_{n_{1}, \ldots, n_{i}}\right)<1 / k\right\}\right)$. Let $P$ be a countably infinite set. We note that $\{0,1\}^{P}$ can be identified with the set of subsets of $P$ by $\nu \in\{0,1\}^{P} \mapsto\{p \in$ $P: \nu(p)=1\}$. We consider the subspace $\operatorname{Tr}$ of $\{0,1\}^{\mathbb{N}^{<\omega}}$ consisting of the trees, i.e., such subsets of $\mathbb{N}<\omega$ which contain $\emptyset,\left(n_{1}\right),\left(n_{1}, n_{2}\right), \ldots,\left(n_{1}, \ldots, n_{k}\right)$ with every element $\left(n_{1}, \ldots, n_{k}\right)$. We say that $T \in \operatorname{Tr}$ is ill-founded $(T \in \mathrm{IF})$ if there exists 
an infinite sequence of natural numbers $n_{1}, n_{2}, \ldots$ such that $\left(n_{1}, \ldots, n_{k}\right) \in T$ for every $k \in \mathbb{N}$. In the opposite case, we say that $T$ is well-founded $(T \in \mathrm{WF})$.

The following lemma is an easy consequence of [1, Theorem 1].

Lemma 2.1. If a Banach space $X$ is not reflexive, then there exist $x_{1}, x_{2}, \ldots$ in $B_{X}$ and a bounded sequence $u_{1}, u_{2}, \ldots$ in $X^{*}$ such that, for every $k, j \in \mathbb{N}$,

$$
u_{k}\left(x_{j}\right) \geq 1 \quad \text { if } k \leq j, \quad u_{k}\left(x_{j}\right)=0 \quad \text { if } k>j .
$$

Proposition 2.2. Let $X$ be a non-reflexive Banach space. Then there is a mapping $\theta: \mathbb{N}<\omega \rightarrow B_{X}$ such that

(i) if $T \in \mathrm{IF}$, then there are distinct $\eta_{1}, \eta_{2}, \cdots \in T$ such that the sequence $\theta\left(\eta_{1}\right), \theta\left(\eta_{2}\right), \ldots$ is convergent, and so $\bigcap_{U \subset T,|U|<\infty} \overline{\mathrm{co}}(\theta(T \backslash U)) \neq \emptyset$,

(ii) if $T \in \mathrm{WF}$, then $\bigcap_{U \subset T,|U|<\infty} \overline{\mathrm{CO}}(\theta(T \backslash U))=\emptyset$.

Proof. Let $x_{1}, x_{2}, \ldots, u_{1}, u_{2}, \ldots$ be as in Lemma 2.1. We define

$$
\theta\left(n_{1}, \ldots, n_{k}\right)=\sum_{i=1}^{k} 2^{-i} x_{n_{i}}
$$

for $\left(n_{1}, \ldots, n_{k}\right) \in \mathbb{N}<\omega$. To prove (i), it is sufficient to realize that, for $n_{1}, n_{2}, \cdots \in$ $\mathbb{N}$, the sequence $\theta(\emptyset), \theta\left(n_{1}\right), \theta\left(n_{1}, n_{2}\right), \ldots$ converges to $\sum_{i=1}^{\infty} 2^{-i} x_{n_{i}}$.

Assume that (ii) does not hold. Let $T \in \mathrm{WF}$ and $a \in \bigcap_{U \subset T,|U|<\infty} \overline{\mathrm{co}}(\theta(T \backslash U))$. The sequence $u_{1}, u_{2}, \ldots$ is bounded, so it is easy to check that the set $\{x \in X$ : $\left.\lim _{k \rightarrow \infty} u_{k}(x)=0\right\}$ is closed. We have

$$
a \in \overline{\mathrm{co}}\left(\theta\left(\mathbb{N}^{<\omega}\right)\right) \subset \overline{\operatorname{sp}}\left\{x_{1}, x_{2}, \ldots\right\} \subset\left\{x \in X: \lim _{k \rightarrow \infty} u_{k}(x)=0\right\},
$$

and so $\lim _{k \rightarrow \infty} u_{k}(a)=0$. We can choose natural numbers $N_{1}, N_{2}, \ldots$ such that

$$
\sum_{i=1}^{l} 2^{i} u_{N_{i}}(a)<1 \quad \text { for } l \in \mathbb{N}
$$

(for example choose $N_{i}$ such that $u_{N_{i}}(a) \leq 2^{-2 i}$ ). The set

$$
R=\left\{\left(n_{1}, \ldots, n_{k}\right) \in T: 1 \leq i \leq k \Rightarrow n_{i} \leq N_{i}\right\}
$$

(where $k$ denotes the length of $\left(n_{1}, \ldots, n_{k}\right)$ ) is finite by König's lemma (cf., [2, Exercise 4.12]). Thus there exists $l \in \mathbb{N}$ such that $l$ is greater than the length of any element of $R$. We are going to prove the following implication:

$$
\left(n_{1}, \ldots, n_{k}\right) \in T \backslash R \quad \Rightarrow \quad \sum_{i=1}^{l} 2^{i} u_{N_{i}}\left(\theta\left(n_{1}, \ldots, n_{k}\right)\right) \geq 1 .
$$

Let $\left(n_{1}, \ldots, n_{k}\right) \in T \backslash R$. Let us realize that $n_{j}>N_{j}$ for some $j \leq \min \{k, l\}$. It is clear in the case that $k \leq l$. If $k>l$ and $n_{j} \leq N_{j}$ for every $j \leq l$, then the sequence $\left(n_{1}, \ldots, n_{l}\right)$ of the first $l$ members of $\left(n_{1}, \ldots, n_{k}\right)$ would be an element of $R$, but its length would be $l$ at the same time, which is impossible. We have

$$
\sum_{i=1}^{l} 2^{i} u_{N_{i}}\left(\theta\left(n_{1}, \ldots, n_{k}\right)\right) \geq 2^{j} u_{N_{j}}\left(\theta\left(n_{1}, \ldots, n_{k}\right)\right)=\sum_{i=1}^{k} 2^{j-i} u_{N_{j}}\left(x_{n_{i}}\right) \geq u_{N_{j}}\left(x_{n_{j}}\right) \geq 1,
$$


and the implication holds. Now, as $R$ is finite,

$$
a \in \bigcap_{U \subset T,|U|<\infty} \overline{\operatorname{co}}(\theta(T \backslash U)) \subset \overline{\mathrm{co}}(\theta(T \backslash R)) \subset\left\{x \in X: \sum_{i=1}^{l} 2^{i} u_{N_{i}}(x) \geq 1\right\},
$$

which is a contradiction with the choice of $N_{1}, N_{2}, \ldots$.

Lemma 2.3. Let $Y$ be an infinite-dimensional normed linear space and $\left(u_{\gamma}\right)_{\gamma \in \Gamma}$ be a system of elements of $Y^{*}$. Let $\left(\delta_{\gamma}\right)_{\gamma \in \Gamma}$ be a system of elements of $(0, \infty]$ such that $\left\{\gamma \in \Gamma: \delta_{\gamma}>\delta\right\}$ is finite for every $\delta>0$ and let $\left(\varepsilon_{\gamma}\right)_{\gamma \in \Gamma}$ be a system of positive numbers. If

$$
g(y)=\max \left\{u_{\gamma}(y)-\varepsilon_{\gamma}\|y\|: \gamma \in \Gamma,\|y\|<\delta_{\gamma}\right\}, \quad y \in Y,\|y\|<\max _{\gamma \in \Gamma} \delta_{\gamma},
$$

then

$$
\partial g(0) \subset \bigcap_{U \subset \Gamma,|U|<\infty} \overline{\operatorname{co}}\left\{u_{\gamma}: \gamma \in \Gamma \backslash U\right\} .
$$

In fact, if $\left\{\gamma \in \Gamma: \varepsilon_{\gamma}>\varepsilon\right\}, \varepsilon>0$, are also finite, then the equality holds. We do not use the inclusion " $\supset$ ", but we prove an analogy of it elsewhere.

Proof. Suppose that $u \in Y^{*} \backslash \bigcap_{U \subset \Gamma,|U|<\infty} \overline{\mathrm{co}}\left\{u_{\gamma}: \gamma \in \Gamma \backslash U\right\}$. We have to prove that $u \notin \partial g(0)$. For some finite $U \subset \Gamma, u$ is not in $\overline{c o}\left\{u_{\gamma}: \gamma \in \Gamma \backslash U\right\}$. By the Hahn-Banach theorem, there exist $F_{0} \in Y^{* *}$ and $\alpha>0$ such that $F_{0}\left(u_{\gamma}-u\right) \geq \alpha$ for every $\gamma \in \Gamma \backslash U$. We can choose $\beta \in\left(0,1 /\left\|F_{0}\right\|\right]$ such that $-\beta F_{0}\left(u_{\gamma}-u\right)<\frac{1}{2} \varepsilon_{\gamma}$ for every $\gamma \in U$. We define $F=-\beta F_{0}, \varepsilon=\min \{\alpha \beta\} \cup\left\{\frac{1}{2} \varepsilon_{\gamma}: \gamma \in U\right\}$. We have $\|F\| \leq 1$ because $\|F\|=\beta\left\|F_{0}\right\| \leq\left(1 /\left\|F_{0}\right\|\right)\left\|F_{0}\right\|=1$. Let us verify that

$$
F\left(u_{\gamma}-u\right)<\varepsilon_{\gamma}-\varepsilon, \quad \gamma \in \Gamma .
$$

If $\gamma \in U$, then $F\left(u_{\gamma}-u\right)=-\beta F_{0}\left(u_{\gamma}-u\right)<\frac{1}{2} \varepsilon_{\gamma}=\varepsilon_{\gamma}-\frac{1}{2} \varepsilon_{\gamma} \leq \varepsilon_{\gamma}-\varepsilon$. If $\gamma \in \Gamma \backslash U$, then $F\left(u_{\gamma}-u\right)=-\beta F_{0}\left(u_{\gamma}-u\right) \leq-\beta \alpha \leq-\varepsilon<\varepsilon_{\gamma}-\varepsilon$.

Now, let $\delta>0$ be given. Since $\left\{G \in Y^{* *}: \delta<\delta_{\gamma} \Rightarrow G\left(u_{\gamma}-u\right)<\varepsilon_{\gamma}-\varepsilon\right\}$ is a neighbourhood of $F$ in the $w^{*}$-topology on $Y^{* *}$, by Goldstine's lemma, there exists $y_{0} \in Y,\left\|y_{0}\right\| \leq 1$, such that $\delta<\delta_{\gamma}$ implies that $\left(u_{\gamma}-u\right)\left(y_{0}\right)<\varepsilon_{\gamma}-\varepsilon$. Since $Y$ is infinite-dimensional, there exists $z \in Y, z \neq 0$, such that $\delta<\delta_{\gamma}$ implies that $\left(u_{\gamma}-u\right)(z)=0$. For an appropriate $\lambda \in \mathbb{R}$, we have $\|y\|=\delta$, where $y=$ $\delta y_{0}+\lambda z$. Let $\gamma \in \Gamma$ be such that $\|y\|<\delta_{\gamma}$. It means that $\delta<\delta_{\gamma}$. We have $\left(u_{\gamma}-u\right)(y)=\left(u_{\gamma}-u\right)\left(\delta y_{0}+\lambda z\right)<\delta\left(\varepsilon_{\gamma}-\varepsilon\right)=\|y\|\left(\varepsilon_{\gamma}-\varepsilon\right)$. Thus, $\|y\|<\delta_{\gamma}$ implies that $\frac{1}{\|y\|}\left(u_{\gamma}(y)-\varepsilon_{\gamma}\|y\|-u(y)\right)<-\varepsilon$. In other words, $\frac{1}{\|y\|}(g(y)-u(y))<-\varepsilon$.

For arbitrary $\delta>0$, we have found $y \in Y,\|y\| \leq \delta$, such that $\frac{1}{\|y\|}(g(y)-u(y))<$ $-\varepsilon$. So $u$ is not a subgradient of $g$ at 0 , and the proof is finished.

Theorem 2.4. Let $X, Y$ be normed linear spaces such that the completion of $Y$ is not reflexive. If $M \subset X$ is a Suslin set, then there exists a Lipschitz function $f$ on $X \oplus_{\infty} Y$ such that, for every $a \in X,(a, 0) \in S(f)$ if and only if $a \in M$.

Proof. Let $A_{n_{1}, \ldots, n_{k}},\left(n_{1}, \ldots, n_{k}\right) \in \mathbb{N}^{<\omega}$, be a system of open subsets of $X$ satisfying (1). We may suppose that, for every $\left(n_{1}, \ldots, n_{k}\right) \in \mathbb{N}<\omega$ and $n_{k+1} \in \mathbb{N}$, $A_{n_{1}, \ldots, n_{k}, n_{k+1}} \subset A_{n_{1}, \ldots, n_{k}}$, i.e., that

$$
T_{a}=\left\{\eta \in \mathbb{N}^{<\omega}: a \in A_{\eta}\right\}
$$


is a tree for every $a \in X$ (we can take $\bigcap_{i=1}^{k} A_{n_{1}, \ldots, n_{i}}$ instead of $A_{n_{1}, \ldots, n_{k}}$ ). We observe that $T_{a} \in \mathrm{IF}$ if and only if $a \in M$.

Now, we are going to use the non-reflexivity of $Y^{*}$. Let $\theta: \mathbb{N}^{<\omega} \rightarrow B_{Y^{*}}$ be as in Proposition 2.2. It follows from (i), (ii) and the observation that

$$
a \in M \quad \Leftrightarrow \quad \bigcap_{U \subset T_{a},|U|<\infty} \overline{\operatorname{co}}\left(\theta\left(T_{a} \backslash U\right)\right) \neq \emptyset
$$

for every $a \in X$. We choose two systems $\left(\delta_{\eta}\right)_{\eta \in \mathbb{N}<\omega}$ and $\left(\varepsilon_{\eta}\right)_{\eta \in \mathbb{N}<\omega}$ of elements of $(0,1)$ such that $\left\{\eta \in \mathbb{N}^{<\omega}: \delta_{\eta}>c\right\},\left\{\eta \in \mathbb{N}^{<\omega}: \varepsilon_{\eta}>c\right\}$ are finite for every $c>0$. For every $\eta \in \mathbb{N}^{<\omega}$, we define

$$
\begin{gathered}
D_{\eta}=\left\{(x, y) \in X \times Y: x \in A_{\eta},\|y\|<\operatorname{dist}\left(x, X \backslash A_{\eta}\right) \text { and }\|y\|<\delta_{\eta} / 2\right\}, \\
E_{\eta}=\left\{(x, y) \in X \times Y: x \notin A_{\eta} \text { or }\|y\| \geq \delta_{\eta}\right\}, \\
f_{\eta}(x, y)= \begin{cases}\theta(\eta)(y)-\varepsilon_{\eta}\|y\| & (x, y) \in D_{\eta}, \\
-2\|y\| & (x, y) \in E_{\eta} .\end{cases}
\end{gathered}
$$

We are going to prove that $f_{\eta}$ is 6-Lipschitz on $D_{\eta} \cup E_{\eta}$. Obviously, $f_{\eta}$ is 6-Lipschitz (in fact, 2-Lipschitz) on $D_{\eta}$ and on $E_{\eta}$. Let $\left(x_{1}, y_{1}\right) \in D_{\eta}$ and $\left(x_{2}, y_{2}\right) \in E_{\eta}$. Since $\left|f_{\eta}\left(x_{1}, y_{1}\right)-f_{\eta}\left(x_{2}, y_{2}\right)\right|=\left|\theta(\eta)\left(y_{1}\right)-\varepsilon_{\eta}\left\|y_{1}\right\|+2\left\|y_{2}\right\|\right| \leq 2\left\|y_{1}\right\|+2\left\|y_{2}\right\|$, it remains to verify that $2\left\|y_{1}\right\|+2\left\|y_{2}\right\| \leq 6\left\|\left(x_{1}, y_{1}\right)-\left(x_{2}, y_{2}\right)\right\|$. If $x_{2} \in A_{\eta}$, then $\left\|y_{2}\right\| \geq \delta_{\eta}$, and thus $2\left\|y_{1}\right\|+2\left\|y_{2}\right\| \leq-6\left\|y_{1}\right\|+4 \delta_{\eta}+6\left\|y_{2}\right\|-4 \delta_{\eta} \leq 6\left\|\left(x_{1}, y_{1}\right)-\left(x_{2}, y_{2}\right)\right\|$. If $x_{2} \notin A_{\eta}$, then $\left\|y_{1}\right\|<\operatorname{dist}\left(x_{1}, X \backslash A_{\eta}\right) \leq\left\|x_{1}-x_{2}\right\|$, and thus $2\left\|y_{1}\right\|+2\left\|y_{2}\right\| \leq$ $4\left\|x_{1}-x_{2}\right\|+2\left\|y_{2}\right\|-2\left\|y_{1}\right\| \leq 6\left\|\left(x_{1}, y_{1}\right)-\left(x_{2}, y_{2}\right)\right\|$.

We recall that the supremum of a non-empty system of $c$-Lipschitz functions is a $c$-Lipschitz function unless it is identically equal to $+\infty$.

Now, $f_{\eta}$ can be extended from $D_{\eta} \cup E_{\eta}$ to $X \times Y$ to be 6 -Lipschitz and to satisfy

$$
f_{\eta}(x, y) \leq \theta(\eta)(y)-\varepsilon_{\eta}\|y\|, \quad(x, y) \in X \times Y
$$

(a 6-Lipschitz extension of $f_{\eta}$ exists by the McShane-Whitney extension theorem (4]); then we can take the minimum of this extension and the function $(x, y) \mapsto$ $\left.\theta(\eta)(y)-\varepsilon_{\eta}\|y\|\right)$. We put

$$
f=\sup \left\{f_{\eta}: \eta \in \mathbb{N}^{<\omega}\right\} .
$$

Obviously, $f$ is 6 -Lipschitz. It remains to prove that, for every $a \in X$,

$$
\bigcap_{U \subset T_{a},|U|<\infty} \overline{\operatorname{co}}\left(\theta\left(T_{a} \backslash U\right)\right) \neq \emptyset \quad \Leftrightarrow \quad(a, 0) \in S(f) .
$$

Let us prove the implication " $\Leftarrow$ ". Let $a \in X$ and $\bigcap_{U \subset T_{a},|U|<\infty} \overline{\operatorname{co}}\left(\theta\left(T_{a} \backslash U\right)\right)=\emptyset$. We consider the function $g$ on $Y$ defined by

$$
g(y)=\max \left(\left\{\theta(\eta)(y)-\varepsilon_{\eta}\|y\|: \eta \in T_{a},\|y\|<\delta_{\eta}\right\} \cup\{-2\|y\|\}\right), \quad y \in Y .
$$

By Lemma 2.3 (applied on $\Gamma=T_{a} \cup\{1\}, \delta_{1}=\infty, \varepsilon_{1}=2, u_{1}=0, u_{\eta}=\theta(\eta)$ for $\left.\eta \in T_{a}\right), \partial g(0) \subset \bigcap_{U \subset T_{a},|U|<\infty} \overline{\operatorname{co}}\left(\theta\left(T_{a} \backslash U\right)\right)$. So $\partial g(0)=\emptyset$. Let us verify that $f_{\eta}(a, \cdot) \leq g$ for every $\eta \in \mathbb{N}^{<\omega}$, and thus $f(a, \cdot) \leq g$. If $\eta \notin T_{a}$, i.e., $a \notin A_{\eta}$, then $f_{\eta}(a, \cdot)=-2\|\cdot\| \leq g$. If $\eta \in T_{a}$ and $\|y\| \geq \delta_{\eta}$, then $(a, y) \in E_{\eta}$, and thus $f_{\eta}(a, y)=$ $-2\|y\| \leq g(y)$ again. If $\eta \in T_{a}$ and $\|y\|<\delta_{\eta}$, then $f_{\eta}(a, y) \leq \theta(\eta)(y)-\varepsilon_{\eta}\|y\| \leq g(y)$. Now, the inequality $f(a, \cdot) \leq g$ is verified. Since $f(a, 0)=g(0)=0$, we get $\partial(f(a, \cdot))(0) \subset \partial g(0)=\emptyset$. Hence, $\partial f(a, 0)=\emptyset$, which proves the implication. 
Let us prove the other implication. Let $a \in X$ and $u \in \bigcap_{U \subset T_{a},|U|<\infty} \overline{\overline{C O}}\left(\theta\left(T_{a} \backslash U\right)\right)$. Let $\varepsilon>0$. Since $u \in \overline{\mathrm{co}}\left\{\theta(\eta): \eta \in T_{a}, \varepsilon_{\eta} \leq \varepsilon / 2\right\}$, there is a finite subset $V$ of $T_{a}$ such that $\varepsilon_{\eta} \leq \varepsilon / 2$ for $\eta \in V$ and $\|u-v\| \leq \varepsilon / 2$ for some $v \in \operatorname{co}(\theta(V))$. We have $f(x, y) \geq f_{\eta}(x, y)=\theta(\eta)(y)-\varepsilon_{\eta}\|y\| \geq \theta(\eta)(y)-(\varepsilon / 2)\|y\|$ for $\eta \in V$ and $(x, y) \in D_{\eta}$. So $f(x, y) \geq v(y)-(\varepsilon / 2)\|y\|$ for $(x, y) \in \bigcap_{\eta \in V} D_{\eta}$. As $V \subset T_{a}$, we have $(a, 0) \in \bigcap_{\eta \in V} D_{\eta}$. Consequently, $f(x, y) \geq u(y)-\varepsilon\|y\|$ on some neighbourhood of $(a, 0)\left(D_{\eta}\right.$ are open because $A_{\eta}$ are open). Since $\varepsilon>0$ was arbitrary, $(x, y) \mapsto u(y)$ is a subgradient of $f$ at $(a, 0)$, and the implication " $\Rightarrow$ " is proved.

Proof of Theorem 1.3. Let the completion of a normed linear space $X$ be nonreflexive. Then $X$ is isomorphic to $\mathbb{R} \oplus_{\infty} Y$, where $Y$ is a subspace of $X$ of codimension 1. The completion of $Y$ is also non-reflexive. A well-known fact says that there is $M \subset \mathbb{R}$, which is Suslin, but not Borel. By Theorem 2.4, there is a Lipschitz function $f$ on $\mathbb{R} \oplus_{\infty} Y$ such that, for every $a \in \mathbb{R},(a, 0) \in S(f)$ if and only if $a \in M$. Since $M$ is not Borel, $S(f)$ is not Borel either.

\section{A BY-PRODUCT}

As a consequence of Proposition 2.2, the non-Borelness of some natural sets of sequences in a non-reflexive space can be shown.

Lemma 3.1. Let $X$ be a non-reflexive Banach space. Then there is a continuous mapping $\Theta: \operatorname{Tr} \rightarrow\left(B_{X}\right)^{\mathbb{N}}$ such that

(i*) if $T \in \mathrm{IF}$, then $\Theta(T)$ has a convergent subsequence,

(ii*) if $T \in \mathrm{WF}$, then $\bigcap_{n=1}^{\infty} \overline{\mathrm{co}}\left\{x_{k}: k \geq n\right\}=\emptyset$.

Proof. First, let $T^{\prime}$ be a fixed infinite well-founded tree. The mapping $T \mapsto T \cup T^{\prime}$ is continuous, and the image of each ill-founded (well-founded) tree is an infinite ill-founded (well-founded) tree. Second, let $\mathbb{N}<\omega$ be ordered to a sequence. The mapping $f:\{T \in \operatorname{Tr}:|T|=\infty\} \rightarrow\left(\mathbb{N}^{<\omega}\right)^{\mathbb{N}}$ induced by the restriction of this ordering to each infinite tree is continuous, and the image of an infinite tree is a sequence of its elements. Let $\theta$ be as in Proposition 2.2. We define

$$
\Theta(T)=\left(\theta\left(f\left(T \cup T^{\prime}\right)(n)\right)\right)_{n \in \mathbb{N}^{\prime}}, \quad T \in \operatorname{Tr} .
$$

Now, $\Theta$ is continuous, and the conditions ( $\left.i^{*}\right)$, (ii*) follow from (i), (ii).

Proposition 3.2. Let $X$ be a non-reflexive Banach space. Then the following sets are not Borel in $\left(B_{X}\right)^{\mathbb{N}}$ :

$$
\begin{aligned}
& A=\left\{\left(x_{1}, x_{2}, \ldots\right) \in\left(B_{X}\right)^{\mathbb{N}}: x_{1}, x_{2}, \ldots \text { has a convergent subsequence }\right\} \\
& B=\left\{\left(x_{1}, x_{2}, \ldots\right) \in\left(B_{X}\right)^{\mathbb{N}}: x_{1}, x_{2}, \ldots \text { has a w-convergent subsequence }\right\} \\
& C=\left\{\left(x_{1}, x_{2}, \ldots\right) \in\left(B_{X}\right)^{\mathbb{N}}: x_{1}, x_{2}, \ldots \text { has a w-cluster point }\right\} \\
& D=\left\{\left(x_{1}, x_{2}, \ldots\right) \in\left(B_{X}\right)^{\mathbb{N}}: \bigcap_{n=1}^{\infty} \overline{\operatorname{co}}\left\{x_{k}: k \geq n\right\} \neq \emptyset\right\} .
\end{aligned}
$$

Proof. Taking $\Theta$ as in Lemma 3.1, we have $\Theta(\mathrm{IF}) \subset A \subset B \subset C \subset D \subset\left(B_{X}\right)^{\mathbb{N}} \backslash$ $\Theta($ WF $)$. Thus, IF $=\Theta^{-1}(A)=\Theta^{-1}(B)=\Theta^{-1}(C)=\Theta^{-1}(D)$, and the well-known fact that IF is not Borel in $\operatorname{Tr}$ (see, e.g., [2]) completes the proof.

\section{ACKNOWLEDGEMENT}

The author would like to thank Petr Holický for helpful discussions and useful remarks. 


\section{REFERENCES}

[1] R. C. James: Characterizations of reflexivity, Studia Math., 23 (1964), 205-216. MR0170192 $(30: 431)$

[2] A. S. Kechris: Classical descriptive set theory, Springer, New York, $1995 . \quad$ MR:1321597 (96e:03057)

[3] O. Kurka: On Borel classes of sets of Fréchet subdifferentiability, Bull. Polish Acad. Sci. Math., 55 (2007), 201-209. MR2346098

[4] E. McShane: Extension of range of functions, Bull. Amer. Math. Soc., 40 (1934), 837-842. MR.1562984

[5] M. Smídek: Measureability of some subsets of spaces of functions (in Czech), Charles University, Prague, 1994.

[6] L. Zajíček: Frechet differentiability, strict differentiability and subdifferentiability, Czechoslovak Math. J., 41 (1991), 471-489. MR1117801 (92j:46081)

Department of Mathematical Analysis, Faculty of Mathematics and Physics, Charles University, Sokolovská 83, 18600 Prague 8, Czech Republid

E-mail address: ondrej.kurka@mff.cuni.cz 